\title{
\begin{tabular}{l|l} 
Mibraries & DSpace@MIT
\end{tabular}
}

\author{
MIT Open Access Articles
}

\section{An Experimentally Tuned Dynamic Model Predicting Cell Migration for Guidance of Sprouting Endothelial Cells}

The MIT Faculty has made this article openly available. Please share how this access benefits you. Your story matters.

Citation: Wood, Levi, and H. Harry Asada. “An Experimentally Tuned Dynamic Model Predicting Cell Migration for Guidance of Sprouting Endothelial Cells." ASME 2011 Dynamic Systems and Control Conference and Bath/ASME Symposium on Fluid Power and Motion Control, Volume 2, October 31- November 2, 2011, ASME, Arlington, Virginia, 2011, pp. 595-601. ( 2011 by ASME

As Published: http://dx.doi.org/10.1115/DSCC2011-6134

Publisher: ASME International

Persistent URL: http://hdl.handle.net/1721.1/118837

Version: Final published version: final published article, as it appeared in a journal, conference proceedings, or other formally published context

Terms of Use: Article is made available in accordance with the publisher's policy and may be subject to US copyright law. Please refer to the publisher's site for terms of use. 


\section{AN EXPERIMENTALLY TUNED DYNAMIC MODEL PREDICTING CELL MIGRATION FOR GUIDANCE OF SPROUTING ENDOTHELIAL CELLS}

\author{
Levi Wood \\ Department of Mechanical Engineering \\ Massachusetts Institute of Technology \\ Cambridge, Massachusetts 02139 \\ E-mail:woodl@mit.edu
}

\author{
H. Harry Asada \\ Department of Mechanical Engineering \\ Massachusetts Institute of Technology \\ Cambridge, Massachusetts 02139 \\ E-mail: asada@mit.edu
}

\begin{abstract}
Endothelial cells (ECs) create a vascular network with a tubular structure in response to growth factors diffused into the gel and interactions with the surrounding environment. Individual cells migrate in response to all of these cues, leading to network pattern formation. This paper presents a dynamic model predicting EC sprout growth that is tuned to time-lapse experimental cell migration data obtained from microfluidic $3 D$ culture. Simple cell migration equations with just a few parameters are formulated and a Maximum Likelihood estimator is used for estimating model parameters from experimental data. The tuned model is used to evaluate the influence of different sprout elongation rates on cell density in the sprout stalk. This quantitative modeling approach will lead to input shaping and feedback control to optimize sprouting metrics such as stalk cell density.
\end{abstract}

\section{INTRODUCTION}

Angiogenesis is the process of growing new blood vessels from existing vessels in the body. It is involved in cancer growth and metastasis [1], wound healing [2,3], embryonic development [4], and is essential for providing perfusion and guidance cues in tissue and organ growth [5]. Understanding how to regulate angiogenic growth will have an important impact in medicinal therapy and tissue engineering application areas.

A growing blood vessel consists of a collection of endothelial cells that work and migrate together from a previously existing vessel into the surrounding matrix scaffold in response to biophysical and biochemical factors. The leading cell, called a "tip cell", leads the way in response to biochemical angiogenic promoters such as Vascular Endothelial Growth Factor (VEGF) among many others [6]. The tip cell secretes matrix metalloproteinases (MMPs) that degrade the matrix and migrate in the direction of positive VEGF gradient $[6,7]$. The cells that follow behind the tip cell, called "stalk cells" typically line the "conduit" that the tip cell has created $[8,9]$ and migrate toward the tip and proliferate to create a continuous connection between the tip and the preexisting blood vessel.

The total sprout growth process involves a multitude of complex chemical and mechanical interactions between the cells, and between the cells and the matrix. In addition, the cells act as agents that make specific phenotypic decisions, including whether they should migrate, proliferate, apoptose, or quiesce [10]. A wide collection of modeling strategies have been proposed in the literature to describe cellular interactions and decisions, as well as total sprout growth. These models have had varied structure, including deterministic continuum models [11], cellular Potts models [12], and stochastic agent-field models [10], to list a few. See [7] for a good summary of mathematical modeling strategies.

While many of the previous modeling works have produced simulated sprouting that was qualitatively similar to experimentally observed angiogenic sprouting, and provided some interesting insights into the role of certain parameters or mechanisms [7], there has been little success in quantitatively prediction the behaviors of individual cells or sprouts.

In this paper, we extend our previous modeling work (see [13]) to quantitatively predict cellular behavior in a developing sprout. We begin by employing microfluidic assays to study 
coordinated cellular behavior in sprout growth and formulate simple cell behavior equations - with just a few parameters based on the experimentally observed behaviors. Next, we formulate a maximum likelihood estimator to estimate the stalk cell equation parameters. We conclude by illustrating that our tuned equations can predict cell migration in developing sprouts and demonstrating in simulation that our tuned equations are useful for planning inputs to control stalk cell density.

\section{EXPERIMENTAL OBSERVATIONS}

\section{Microfluidic Angiogenesis Assay}

Our aim is to formulate equations that predict experimentally observed cell migration in angiogenic sprout growth. We observe growth in 3D microfluidic assays [14]. As shown in Fig. 1, the assay consists of a central region filled with a type I collagen gel matrix. Fluidic flow channels enable application of angiogenic growth factors, such as VEGF, in the cell culture media on either side of the gel matrix. This allows us to apply concentrations and gradients as control inputs.

We begin the experiment by seeding endothelial cells (ECs) into channel B as shown, which form a confluent monolayer on the collagen wall, and channel top and bottom, over $24 \mathrm{hrs}$. After $24 \mathrm{hrs}$, we apply a high concentration of VEGF in channel A (typically $40 \mathrm{ng} / \mathrm{mL}$ ) and a lower concentration in channel B (typically $20 \mathrm{ng} / \mathrm{mL}$ ). The VEGF gradient stimulates sprout growth as shown in the confocal fluorescent images of a sprout with a lumen in Fig. 2. In the figure, the bright blue ovals are live-cell stained nuclei (Hoechst 33342; Invitrogen Cat. No. H1399) and the distributed green signal is the cytosolic live CellTracker CMFDA (Invitrogen Cat. No. C7025).

Observing cell migration over time gives important insight into the dominant cell behaviors. Fig. 2 shows cells 1 and 2 migrating on the outer edge of the conduit forming the lumen, rather than in the middle. Fig. 3 shows another sprout with cells migrating single file along a narrow conduit. Here, the red stain is live CellTracker CMTPX (Invitrogen Cat. No. C34552). As indicated in Figs. 3B and 3C, we have observed the migration and recorded the cell nuclear locations over $15 \mathrm{hr}$ while the sprout grew. The data show that the tip migrates forward and the stalk cells follow behind it in the path, or conduit, that it left in the matrix gel. In addition, while the two stalk cells were originally bunched up in the beginning of the experiment, they quickly attempted to move apart so that they were not too close together. They may have initially been close together due to a proliferation.

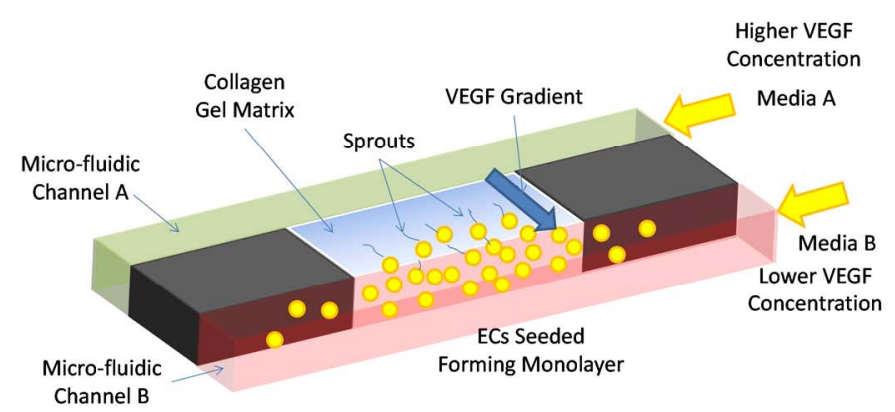

FIGURE 1. MICROFLUIDIC ANGIOGENESIS ASSAY

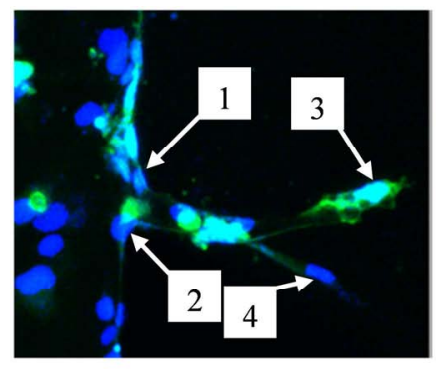

$7.5 \mathrm{hr}$



$9.5 \mathrm{hr}$
FIGURE 2. TIME LAPSE IMAGES OF CELL MIGRATION IN A LUMEN

\section{Key Observations in Cell Migration}

Based on our experimental data, we have observed several key features of cell migration in sprout growth which must be incorporated into the migration equations. These features are discussed in great detail in our previous work [13], so we will list them only briefly here:

- A single tip cell leads the sprout elongation, cleaving the matrix, and leaving a "conduit" behind it (see Fig. 3).

- Multiple stalk cells follow behind the tip and migrate on the surface of the conduit (see Fig. 2).

- Adjacent cells are mechanically coupled; if the cells move too far apart, the sprout can fail by breaking apart (see [13]).

In the following sections, we will briefly construct equations that are consistent with these experimental observations, then we will investigate how to tune the model structure and parameters to quantitatively predict the stalk cell migration trajectories shown in Fig. 3. 

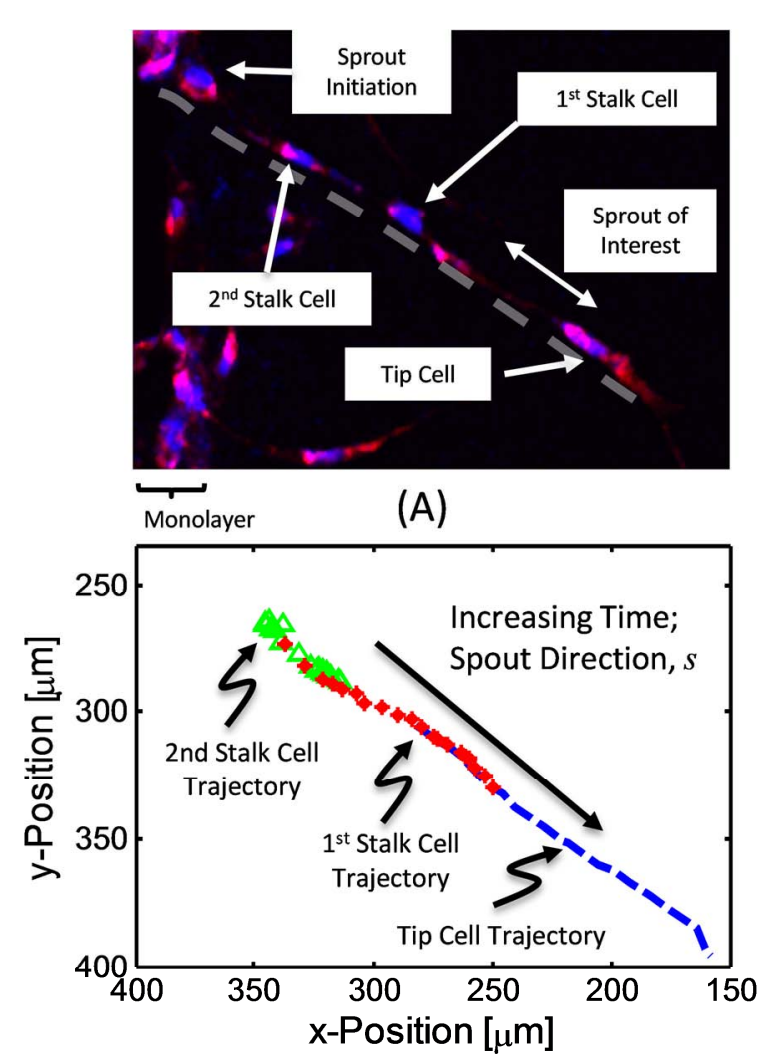

(B)

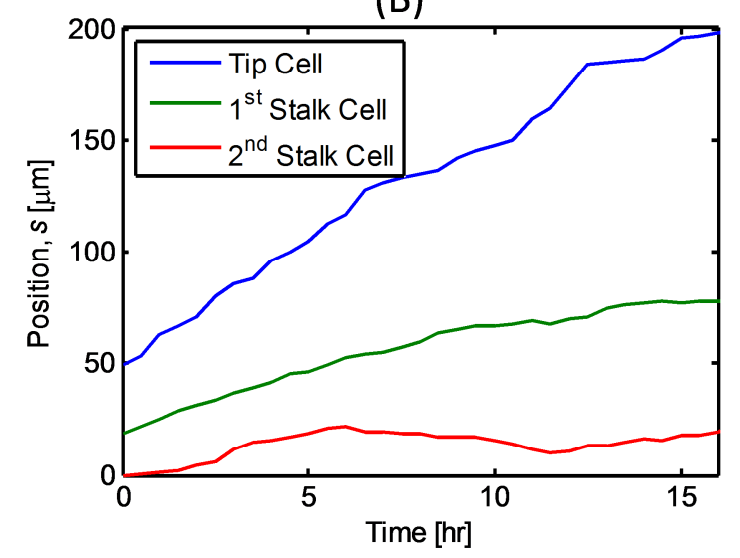

(C)

FIGURE 3. TRACKING COORDINATED CELL

MIGRATION OVER TIME. (A) IMAGE OF SPROUT AT END;

(B) CELL TRAJECTORIES; (C) DISTANCE TRAVELED VS. TIME

\section{DYNAMIC EQUATION FORMULATION}

\section{Tip cell dynamics}

Tip cell migration is a 3-D dynamical process guided by several factors. Most prominent and well studied is the gradient of chemo attractant, VEGF. A tip cell is capable of detecting the gradient of VEGF by extending filopodia in diverse directions. In the following dynamic formulation, only VEGF is considered as an exogenous growth factor that guides the migration of the tip cell. Let $u_{t}(\mathbf{x}) \in \mathfrak{R}^{1}$ be the concentration of VEGF at coordinates $\mathbf{x}$ in the matrix field and $\nabla u_{t}(\mathbf{x})$ be the gradient of VEGF concentration at $\mathbf{x}$. The tip cell velocity is directed in the positive VEGF gradient direction:

$$
\begin{aligned}
& \mathbf{v}_{t+1}^{t i p}=\left\{\begin{array}{cc}
\mathbf{w}_{t}^{t i p} & ;\left|\nabla u_{t}\right|=0 \\
h_{1}\left(\nabla u_{t} ; a_{1}, d_{1}\right) \cdot \frac{\nabla u_{t}}{\left|\nabla u_{t}\right|}+\mathbf{w}_{t}^{t i p} ; & \left|\nabla u_{t}\right| \neq 0
\end{array}\right. \\
& \mathbf{x}_{t+1}^{t i p}=\mathbf{x}_{t}^{t i p}+(\Delta t) \mathbf{v}_{t}^{t i p}
\end{aligned}
$$

where $\mathbf{w}_{t}^{\text {tip }} \in \mathfrak{R}^{3}$ is an uncorrelated noise with zero mean values and covariance $\mathbf{S}$, and $h_{1}\left(\nabla u_{t}, a_{1}, d_{1}\right)$ is a scalar function that saturates at $a_{1}$ :

$$
h_{1}\left(\nabla u_{t}, a_{1}, d_{1}\right)=a_{1}\left[1-\exp \left(-\left|\nabla u_{t}\right| / d_{1}\right)\right]
$$

Parameters $a_{1}$ and $d_{1}$ as well as covariance $\mathbf{S}$ are to be identified based on experimental data. The scalar function $h_{1}\left(\nabla u_{t}, a_{1}, d_{1}\right)$ is a nonlinear function relating the tip velocity to the gradient of VEGF.

\section{Stalk cell dynamics}

There are several important forces influencing stalk cell migration as given by:

$$
\begin{aligned}
& \mathbf{v}_{t+1}^{i}=\mathbf{v}_{t}^{i}(1-b)+a_{2} \nabla q_{t}-\sum_{j \neq i} \mathbf{f}\left(\mathbf{x}_{t}^{j}-\mathbf{x}_{t}^{i}\right)+\mathbf{R}\left(\mathbf{x}_{t}^{i}\right)+\mathbf{w}_{t}^{\text {stalk }} \\
& \mathbf{x}_{t+1}^{i}=\mathbf{x}_{t}^{i}+(\Delta t) \mathbf{v}_{t}^{i}
\end{aligned}
$$

where the $1^{\text {st }}$ term on the right side of the equation is an autoregressive term, the second term is hypothetical cue released by the tip cell that directs stalk cell migration, the third term takes into account cell-cell interference and connectivity, $\mathbf{R}\left(\mathbf{x}_{t}^{i}\right)$ is a restoring force that forces the stalk cells to migrate along the conduit wall, and $\mathbf{w}_{t}^{\text {stalk }}$ is a white noise term.

We consider the following simple dynamic model for generating $q_{t}(\mathbf{x})$ :

$$
q_{t+1}(\mathbf{x})=\alpha \cdot q_{t}(\mathbf{x})+\exp \left(-\frac{\left|\mathbf{x}-\mathbf{x}_{t}^{t i p}\right|}{d_{2}}\right)
$$

where $\alpha$ is the chemoattractant decay rate, $0<\alpha<1$, and $d_{2}$ is the distance scale of the cue released by the tip cell, which would be related to a diffusion coefficient if the cue were a diffusible species.

The cellular interaction term incorporates cell-cell interference, by repelling cells that are too close together. In addition, it attracts cells that are at an intermediate distance, 
where they may be adhered to each other via cell-cell junctions and attract one another when they are too far apart.

The cell mechanics involved in cell-cell junctions and associated forces are extremely complex. For system identification purposes, we parameterize the interaction forces using a smooth force curve with the following form:

$\mathbf{f}\left(\mathbf{x}_{t}^{j}-\mathbf{x}_{t}^{i}\right)=\left\{\begin{array}{cc}c_{1} \cdot \operatorname{sinc}\left(\frac{|\Delta \mathbf{x}|}{\gamma_{1}}\right) \frac{\Delta \mathbf{x}}{|\Delta \mathbf{x}|}, & 0<|\Delta \mathbf{x}| \leq \gamma_{1} \\ c_{2} \cdot \operatorname{sinc}\left(\frac{|\Delta \mathbf{x}|-\gamma_{1}+\gamma_{2}}{\gamma_{2}}\right) \frac{\Delta \mathbf{x}}{|\Delta \mathbf{x}|}, & \gamma_{1}<|\Delta \mathbf{x}| \leq \gamma_{1}+\gamma_{2} \\ 0, & \text { otherwise }\end{array}\right.$

where $\quad \operatorname{sinc}(x)=\sin (\pi x) / \pi x \quad$ and $\quad \Delta \mathbf{x}=\mathbf{x}_{t}^{j}-\mathbf{x}_{t}^{i} \quad$ for $j \neq i, 1 \leq i, j \leq N$. Eq. (5) is a convenient, piecewise and smooth function with value 0 at $|\Delta \mathbf{x}|=\gamma_{1}, \gamma_{1}+\gamma_{2}$, and it provides a maximum repulsive force at $x=0$. In practice, we have found this form to describe the data, but another smooth form, including a polynomial may also be convenient.

\section{Matrix dynamics}

The collagen gel matrix is degraded mostly by MMP, a protease released by a tip cell. Let $C(\mathbf{x}, t)$ be the concentration of MMP at coordinates $\mathbf{x}$ at time $t$, and $Q$ be the rate at which MMP is produced by a single tip cell. Assuming no interstitial flow, the MMP released by a tip cell diffuses to the local gel matrix, governed by the following diffusion dynamics[10]:

$$
\frac{\partial C(\mathbf{x}, t)}{\partial t}=D \nabla^{2} C(\mathbf{x}, t)+Q \delta\left(\mathbf{x}-\mathbf{x}_{t}^{t i p}\right)
$$

where $D$ is a diffusion coefficient, and $\delta(\bullet)$ is the delta function, which takes 0 other than the tip cell location: $\mathbf{x}=\mathbf{x}_{t}^{t i p}$. As the tip cell migrates, it releases MMP from a different location and in consequence the MMP concentration exhibits a unique distribution depending on the time trajectory of the tip cell.

The MMP degrades the gel matrix by cleaving the cross links of gel fibers. This lowers the "integrity" of the gel matrix, allowing the tip cell to penetrate the gel matrix. Let $I(\mathbf{x}, t)$ be the integrity of gel matrix at coordinates $\mathbf{x}$ and time $t$.

$$
\frac{\partial I(\mathbf{x}, t)}{\partial t}=-k C(\mathbf{x}, t) I(\mathbf{x}, t)
$$

where $k$ is the rate at which the gel matrix is cleaved by MMP.

\section{TUNING THE MODEL}

\section{Maximum Likelihood Estimation}

In this work, we are focused on estimating the parameters in our stalk migration equations from experimental data. Assuming that the covariance of the Gaussian noise $\mathbf{w}_{t}^{\text {stalk }}$ is isotropic, $\sigma_{\text {stalk }}^{2} \mathbf{I}$, the parameters to estimate are $\theta_{\text {stalk }}=\left[b, a_{2}, d_{2}, \alpha, c_{1}, c_{2}, \gamma_{1}, \gamma_{2}, \sigma_{\text {stalk }}\right]^{T}$, where $b$ is viscous damping, $a_{2}, d_{2}$, and $\alpha$ are associated with the cue intensity, and $c_{1}, c_{2}$, and $\gamma$ are involved in cell-cell interaction forces.

Eliminating the uncorrelated noise term $\mathbf{w}_{t}^{\text {stalk }}$, the velocity predictor is given by

$$
\begin{aligned}
\hat{\mathbf{v}}_{t+1}^{i}\left(\mathbf{v}_{t}^{i} ; \hat{\theta}_{\text {stalk }}\right)= & \mathbf{v}_{t}^{i}(1-\hat{b})+\hat{a}_{2} \nabla q_{t}\left(\mathbf{x}_{t}^{i} ; \hat{\alpha}, \mathbf{x}_{\tau}^{t i p}, 0 \leq \tau \leq t\right) \\
& -\sum_{j \neq i} \mathbf{f}\left(\mathbf{x}_{t}^{j}-\mathbf{x}_{t}^{i} ; \hat{c}_{1}, \hat{c}_{2}, \hat{\gamma}_{1}, \hat{\gamma}_{2}\right)+\hat{\mathbf{R}}\left(\mathbf{x}_{t}^{i}\right)
\end{aligned}
$$

Note that the reaction forces $\hat{\mathbf{R}}\left(\mathbf{x}_{t}^{i}\right)$ are normal to the collagen wall and are selected such that $\hat{\mathbf{v}}_{t+1}^{i}\left(\mathbf{x}_{t}^{i}\right) \bullet n\left(\mathbf{x}_{t}^{i}\right)=0$, where $n\left(\mathbf{x}_{t}^{i}\right)$ is a unit vector normal to the conduit wall and pointing inward. Therefore, we estimate the parameters based on the velocity components that are parallel to the wall and normal to $n\left(\mathbf{x}_{t}^{i}\right)$. Note that we do not measure the conduit boundary from the current experiments. However, it is possible to estimate the conduit wall location using the matrix degradation dynamics in Eqs. (6) and (7).

The prediction error is given by the discrepancy from the actual measurement $\mathbf{v}_{t+1}^{\text {stalk }}$

$$
\phi(t, \theta)=\mathbf{v}_{t+1}^{\text {stalk }}-\hat{\mathbf{v}}_{t+1}^{\text {stalk }}\left(\mathbf{v}_{t}^{i} ; \hat{\theta}_{\text {stalk }}\right)
$$

Assuming that the model structure is correct, it follows from the stalk cell dynamic model, Eq. (3), that the probability distribution of the prediction error $\phi\left(t, \theta_{0}\right)$ is Gaussian with zero mean values for the correct parameter distribution $\theta_{0}$, since the prediction error comes from the uncorrelated noise $\mathbf{w}_{t}^{\text {stalk }}$

$$
p_{\Phi}\left(\phi(t) ; \theta_{0}\right) \sim N(0, \mathbf{S})
$$

where $\mathbf{S}$ is the error covariance of noise $\mathbf{w}_{t}^{t i p}$. This implies that the prediction error is independent with respect to the observation, $i$, and the Maximum Likelihood Estimate of the parameter vector is then given by 


$$
\begin{aligned}
\hat{\theta}_{\text {tip }}^{M L E}(M) & =\arg \max _{\theta} \prod_{i=1}^{M} \frac{1}{2 \pi|\mathbf{S}|^{1 / 2}} \\
& \bullet \exp \left(-\frac{1}{2} \phi(t, \theta)^{T} \mathbf{S}^{-1} \phi(t, \theta)\right) \\
& =\arg \min _{\theta}\left(\sum_{i=1}^{M} \phi(t, \theta)^{T} \mathbf{S}^{-1} \phi(t, \theta)+\ln |\mathbf{S}|\right)
\end{aligned}
$$

where $M$ is the total number of stalk cell migration velocity observations taken from all stalk cells during the experiment.

\section{Reducing the Number of Parameters to Tune}

As we found previously in [13], it is not possible to estimate all of the 9 stalk cell parameters by tracking migration velocities alone - the parameters are not persistently excited by the data. This means that some of the parameters are unnecessary to capture the cell dynamics and are either redundant or the migration model is insensitive to them. Therefore, it is important to determine which parameters need to be tuned, which can be estimated from other experiments, and which can be eliminated outright.

The experimental stalk cell speed data (see Fig. 4), shows that the cell migration rate can change by more than a factor of two and even change direction between the 30min samples. This behavior is consistent with the $\sim 30 \mathrm{~min}$ persistence time found in endothelial cell motion on a glass substrate in [15]. Therefore, the autoregressive term in Eq. (3) will be small and we neglect it.

The autoregressive term is the only one we have been able to neglect. However, the cue decay rate, $\alpha$, will be a property of the physical process governing its interaction with the matrix (as will $d_{2}$ ). We have found that we are able to fix $\alpha=0.8$ and estimate the remaining parameters to match the data. This approach is necessary to identify the remaining parameters.

\section{Estimating Stalk Cell Parameters from Experiment}

To estimate the model parameters, we implement the cell migration equations in simulation. The simulation consists of a 100x100x100 grid of unit size $2 \mu \mathrm{m}$. We begin with a monolayer of lined up stalk cells on the left side and a tip cell leading the sprout (see Fig. 5). An initial sprout conduit is carved out and two stalk cells are placed at their respective experimentally observed locations. Next, the simulated velocity is computed for each of the stalk cells according to Eq. (3).

Since our aim is to estimate the stalk cell migration equation parameters, we use the observed tip cell location as a system input. During each time step, we place the simulated stalk cells at the experimentally observed location and compute the error between experimentally observed speed and predicted speed according to Eq. (9). Comparing against all of the experimental observations, we can take $\alpha=0.8$ and

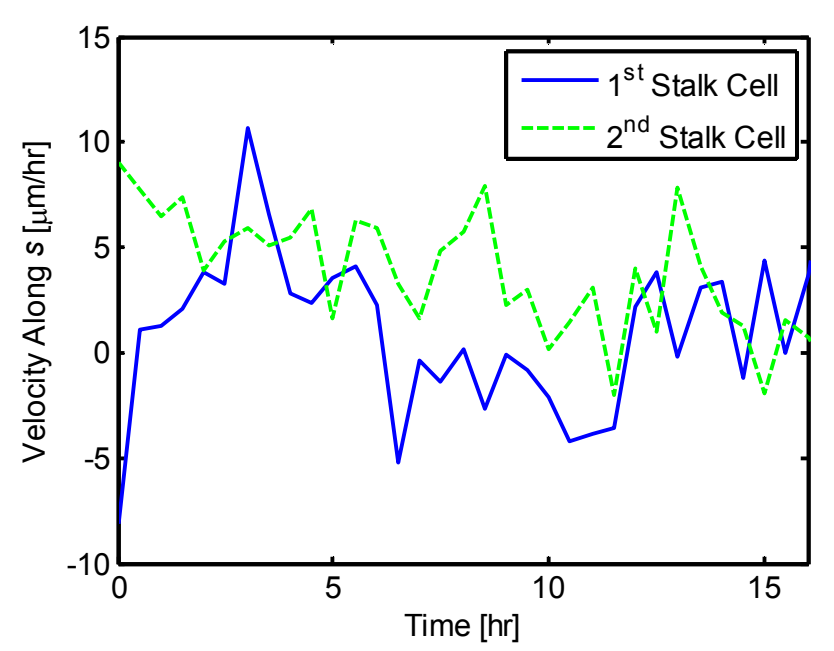

FIGURE 4. VELOCITY OF STALK CELLS ALONG THE SPROUTING AXIS

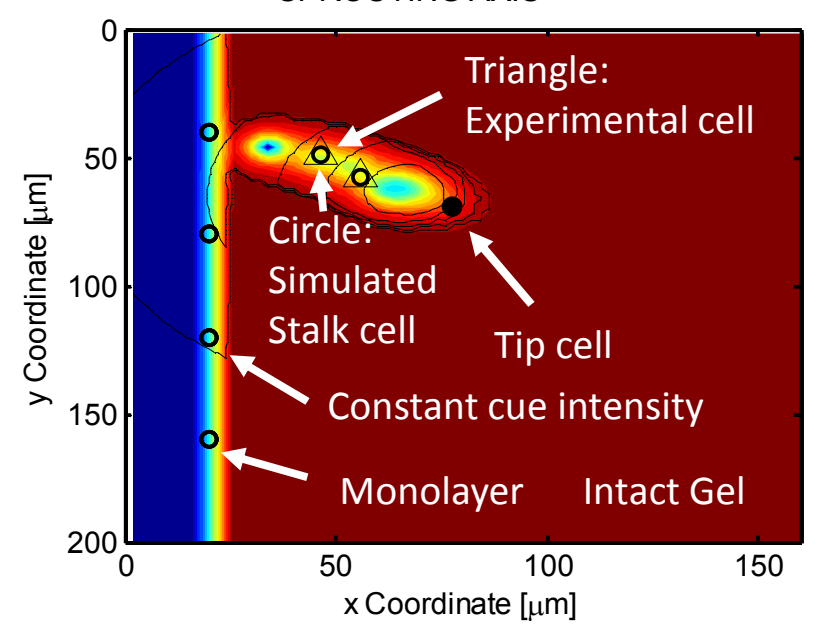

FIGURE 5. SPROUTING SIMULATION INITIALIZED TO EXPERIMENTAL SPROUT

(neglecting the autoregressive term) compute the MLE, according to Eq. (11), which yields Table 1. Note that since the cue mechanism is not specified, we take $q\left(\mathbf{x}_{t}^{i}\right)$ to be unitless.

Using these parameters, we can simulate the stalk cell trajectories during the entire simulation, as shown in Fig. 6. Though the fit isn't perfect, we do a reasonable job of capturing the observed migration trajectories.

\section{TABLE I. ESTIMATED PARAMETER VALUES}

\begin{tabular}{ccccccccc}
\hline Param. & $a_{2}$ & $d_{2}$ & $\gamma_{1}$ & $\gamma_{2}$ & $c_{1}$ & $c_{2}$ & $\alpha$ & $\sigma_{\text {stalk }}$ \\
Units & $\mu \mathrm{m}^{2} / \mathrm{hr}$ & $\mu \mathrm{m}$ & $\mu \mathrm{m}$ & $\mu \mathrm{m}$ & $\mu \mathrm{m} / \mathrm{hr}$ & $\mu \mathrm{m} / \mathrm{hr}$ & - & $\mu \mathrm{m} / \mathrm{hr}$ \\
Value & 5.00 & 79.90 & 29.38 & 32.56 & 4.80 & 4.00 & 0.80 & 1.60 \\
\hline
\end{tabular}




\section{DISCUSSION}

Our modeling scheme is not mechanistic, and it incorporates imperfect hypothetical modeling structures. However our approach is valuable because it is simple enough that we can tune the model parameters to quantitatively describe the system dynamics, which gives us the capability to predict future cellular migration in response to multiple stimuli to determine the best choice to obtain a desired sprouting outcome.

One candidate approach to manipulating sprout growth is to manipulate the gradient of VEGF to manipulate the migration rate of the tip cell, as indicated in Eq. (1). Therefore, it is important to understand how manipulating the tip cell's speed will influence stalk cell migration. Our tuned model provides the necessary means of investigating, and optimizing the tip cell speed trajectory to obtained desired stalk cell migration, including the number of stalk cells in the sprout. For example, Fig. 7 shows that slowing down the tip cell migration

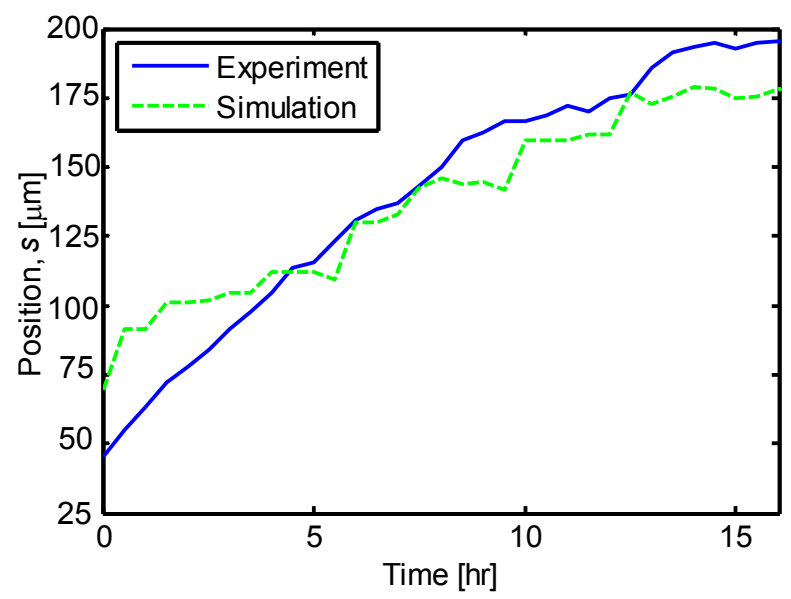

(A)

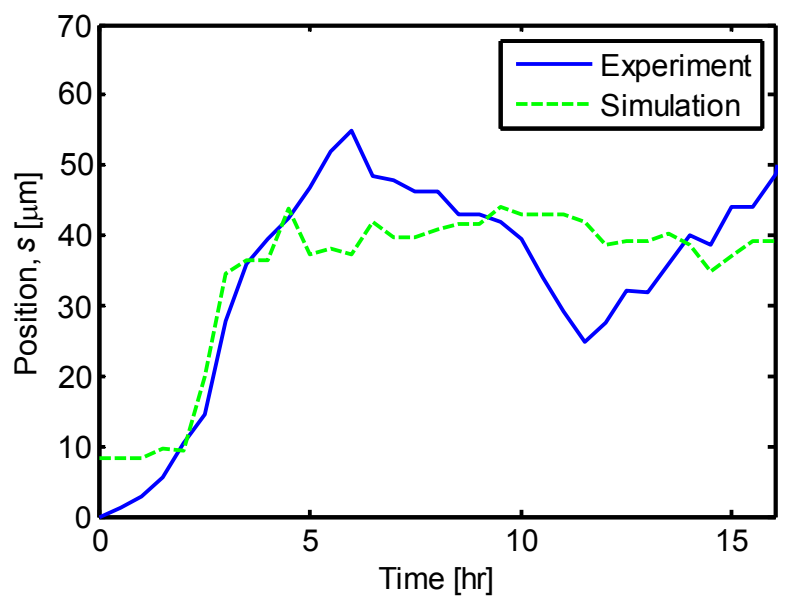

(B)

FIGURE 6. EXPERIMENT AND SIMULATION FOR (A) $1^{\text {ST }}$ STALK CELL; (B) $2^{\mathrm{ND}}$ STALK CELL

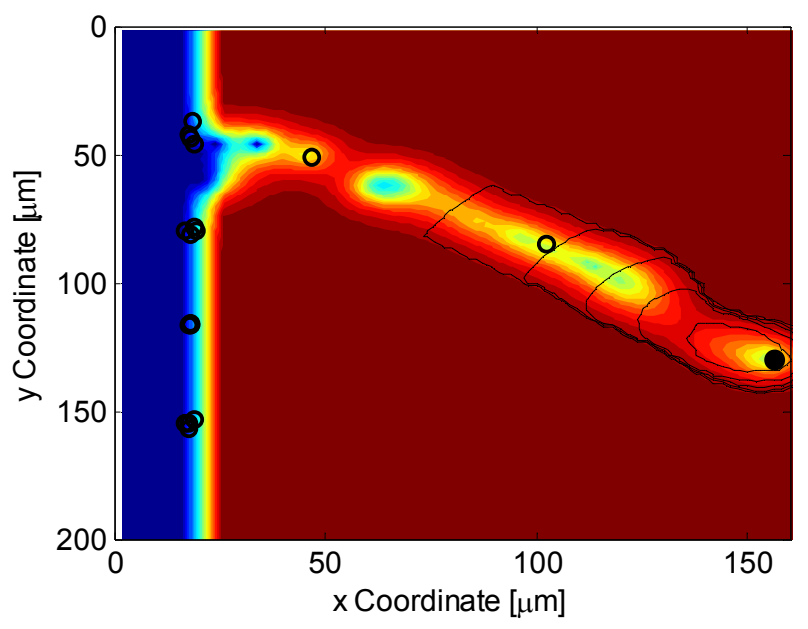

(A)

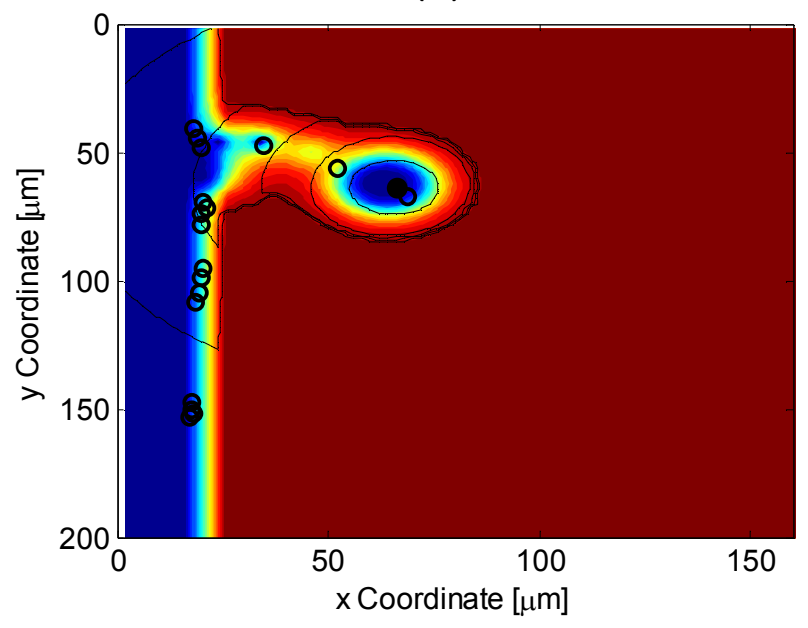

(B)

FIGURE 7. SIMULATED STALK CELL SPACING WITH (A) FAST MOVING TIP CELL; (B) SLOW MOVING TIP CELL

rate will allow more stalk cells to migrate into the sprout, which will limit failure due to breakage of cell-cell junctions.

\section{CONCLUSION}

Our quantitative modeling framework provides a unique capability to predict cellular migration in terms of cellular behaviors observed via live cell microscopy alone. This approach trades off mechanistic process understanding found in prior work for a simple model structure with relatively few parameters that can be estimated from observed migration data.

Our initial formulation had too many parameters to tune from the data. By systematically modifying the equations - and eliminating a parameter - we were able to capture the mean stalk cell migration dynamics. By estimating the equation parameters via a ML estimator, we were able to predict the 
observed migration behaviors and the dynamics of future sprout elongation.

As shown in simulation, the tuned model will allow us to predict the influence on sprout formation of accelerating the tip cell migration by, application of higher gradients of VEGF, and allow us to manipulate system inputs to, for example, maximize migration rate, while maintaining high sprout survivability rate. This modeling approach will lead to quantitative prediction and active control of vascular network growth, which will have important impact in tissue engineering and regenerative medicine.

\section{REFERENCES}

[1] Carmeliet, P., and Jain, R. K., 2000, "Angiogenesis in Cancer and Other Diseases," Nature, 407(6801) pp. 249257.

[2] Arnold, F., and West, D. C., 1991, "Angiogenesis in Wound Healing," Pharmacology \& Therapeutics, 52(3) pp. 407422.

[3] Tonnesen, M. G., Feng, X., and Clark, R. A. F., 2000, "Angiogenesis in wound healing," Journal of Investigative Dermatology Symposium Proceedings, Anonymous Nature Publishing Group, 5, pp. 40-46.

[4] Breier, G., 2000, "Angiogenesis in Embryonic Development--a Review," Placenta, 21pp. S11-S15.

[5] Kaully, T., Kaufman-Francis, K., Lesman, A., 2009, "Vascularization: The Conduit to Viable Engineered Tissues," Tissue Engineering Part B: Reviews, 15(2) pp. 159-169.

[6] Gerhardt, H., 2008, "VEGF in Development,"Springer New York, pp. 68-78, Chap. 6.

[7] Mantzaris, N. V., Webb, S., and Othmer, H. G., 2004, "Mathematical Modeling of Tumor-Induced Angiogenesis," Journal of Mathematical Biology, 49(2) pp. 111-187.

[8] Stratman, A. N., Saunders, W. B., Sacharidou, A., 2009, "Endothelial Cell Lumen and Vascular Guidance Tunnel Formation Requires MT1-MMP-Dependent Proteolysis in 3-Dimensional Collagen Matrices," Blood, 114(2) pp. 237247.

[9] Chun, T. H., Sabeh, F., Ota, I., 2004, "MT1-MMPDependent Neovessel Formation within the Confines of the Three-Dimensional Extracellular Matrix," Journal of Cell Biology, .

[10] Das, A., Lauffenburger, D., Asada, H., 2010, "A Hybrid Contimuum-Discrete Modeling Approach to Predicting and Controlling Angiogenesis: Analysis of Combinatorial Gorwth Factor and Matrix Effects on Vessel Sprouting Morphology," Accepted for Publication in Phil.Trans.A, .

[11] Levine, H. A., Sleeman, B. D., and Nilsen-Hamilton, M., 2000, "A Mathematical Model for the Roles of Pericytes and Macrophages in the Initiation of Angiogenesis. I. the Role of Protease Inhibitors in Preventing Angiogenesis," Mathematical Biosciences, 168(1) pp. 77-115.

\section{ACKNOWLEDGMENTS}

The authors would like to thank Roger Kamm for many fruitful discussions and suggestions that have made this work possible.

This material is based on work supported in part by the Emerging Frontiers in Research and Innovation Program of the National Science Foundation under grant number NSF EFRI0735997. This work is also supported by the Singapore-MIT Alliance for Research and Technology, Bio-Systems and Micromechanics IRG.

[12] Plank, M., Sleeman, B., and Jones, P., 2004, "A Mathematical Model of Tumour Angiogenesis, Regulated by Vascular Endothelial Growth Factor and the Angiopoietins," Journal of Theoretical Biology, 229(4) pp. 435-454.

[13] Wood, L., Kamm, R., and Asada, H., "Stochastic Modeling and Identification of Emergent Behaviors of an Endothelial Cell Population in Angiogenic Pattern Formation," The International Journal of Robotics Research, .

[14] Chung, S., Sudo, R., Mack, P. J., 2009, "Cell Migration into Scaffolds Under Co-Culture Conditions in a Microfluidic Platform," Lab on a Chip, 9(2) pp. 269-275.

[15] Kouvroukoglou, S., Dee, K. C., Bizios, R., 2000, "Endothelial Cell Migration on Surfaces Modified with Immobilized Adhesive Peptides," Biomaterials, 21(17) pp. 1725 . 